\title{
Avaliação da Autoeficácia e da Memória em Idosos: Uma Análise Exploratória
}

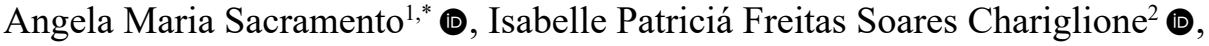 \\ Gislane Ferreira de Melo $^{1}$ (D), \& Carmen Jansen de Cárdenas ${ }^{1}$ (D) \\ ${ }^{1}$ Universidade Católica de Brasília, Brasília, DF, Brasil \\ ${ }^{2}$ Universidade de Brasília, Brasília, DF, Brasil
}

\begin{abstract}
RESUMO - Trata-se de um estudo exploratório, transversal e quantitativo, cujo objetivo foi verificar as alterações da memória e da autoeficácia em 110 idosos, com média de 70,5 anos. Foram utilizados um questionário sociodemográfico, um teste de percepção subjetiva de memória, o questionário de memória Prospectiva e Retrospectiva e a Lista de Aprendizagem Auditivo-Verbal de Rey. Os idosos foram avaliados em um único momento, individualmente, por cerca de 60 minutos. Os resultados do teste de memória apresentaram-se dentro dos padrões esperados para a idade e a escolaridade, com leve desempenho inferior na memória de trabalho e percepção negativa da autoeficácia da memória (82,70\%). Tal desempenho foi associado às queixas subjetivas de memória $(99,10 \%)$, o que pode ser explicado por paradigmas socioculturais atrelados negativamente ao processo de envelhecer.
\end{abstract}

PALAVRAS-CHAVE: Memória, Autoeficácia, Idosos

\section{Self-Efficacy and Memory Assessment in Elderly: An Exploratory Analysis}

\begin{abstract}
It is an exploratory, cross-sectional and quantitative study whose objective was to verify memory and self-efficacy alterations in 110 older adults, with a mean of 70.50 years. We used a sociodemographic questionnaire, a subjective memory perception test, the Prospective and Retrospective Memory Questionnaire, and The Rey AuditoryVerbal Learning Test. The seniors were evaluated in a single moment, individually, for about 60 minutes. The results of the memory test were within the expected standards for age and schooling, with a slightly inferior performance in working memory and negative perception of memory self-efficacy $(82.70 \%)$. Such performance was associated with subjective memory complaints $(99.10 \%)$, which can be explained by socio-cultural paradigms negatively linked to the aging process.
\end{abstract}

KEYWORDS: Memory, Self-Efficacy, Aged

\section{INTRODUÇÃO}

O aumento da longevidade é uma transformação demográfica mundial. Inúmeros fatores contribuíram para esse aumento de expectativa de vida, dentre eles a queda na taxa da fecundidade, a diminuição da mortalidade infantil, as melhorias das condições de saneamento e os avanços da medicina e da tecnologia (Cromartie, 2020; Miranda et al., 2016; Ribeiro et al., 2018).
O processo de envelhecimento apresenta um declínio gradual de diversas funções, dentre elas as cognitivas. O declínio da memória é o que mais se destaca entre as principais queixas da população em geral, pois compromete as tarefas relativas às atividades instrumentais da vida diária (Belleville et al., 2018; Melo et al., 2017). A manutenção da cognição é um componente importante da

*E-mail: sacrapesquisa@gmail.com

- Submetido: 17/05/2018; Revisado: 01/02/2020; Aceito: 19/04/2020. 
qualidade de vida e da longevidade na velhice, uma vez que o declínio cognitivo pode estar associado a desconforto pessoal, perda de autonomia e aumento dos custos sociais (Cançado et al., 2013).

Segundo Baumgart et al. (2015), as alterações cognitivas nos idosos não são estáticas e unitárias, sendo que algumas habilidades declinam mais rapidamente do que as outras, ressaltando que existem habilidades que se mantêm estáveis (linguagem, conhecimento geral e julgamento) ao longo de toda a vida. As alterações cognitivas sofrem relação direta de duas variáveis, qualidade e quantidade da estimulação, as quais o idoso foi disposto ao longo de sua vida.

O declínio da capacidade cognitiva e, em especial, da habilidade da memória pode ser observado também no processo do envelhecimento ativo, isto é, mesmo sem diagnóstico de demências. Esse declínio ocorre em decorrência de fatores genéticos, biológicos, educacionais, sociais e de estilo de vida, que variam de pessoa para pessoa (Burke et al., 2018; Nespollo et al., 2017; Tomaszewski Farias et al., 2018). Outra característica é que os idosos podem apresentar dificuldade tanto no processo de codificação da informação quanto no resgate do material memorizado. Em relação ao resgate, a principal dificuldade acontece quando a evocação é livre, sem uso de pistas (Chariglione, 2014; Nespollo et al., 2017).

$\mathrm{Na}$ memória de curto prazo, as maiores queixas relacionam-se à memória de trabalho, principalmente na redução dos recursos de processamento, e às falhas no mecanismo de inibição de informações supérfluas (Boller et al., 2017; Porto \& Nitrini, 2014). Ou seja, no momento da seleção da informação, há um processo deficitário. Por consequência, o processamento da informação ocorre de forma mais lentificada, na qual há uma competição das informações necessárias com as irrelevantes (Yassuda \& Abreu, 2013; Yassuda et al., 2013).

Outro aspecto da memória de trabalho é a redução no processo de armazenamento, o que interfere na capacidade de retenção de novas informações (Mascarello, 2013). Portanto, a seleção de estímulos deficitários, associada à diminuição da capacidade de armazenamento das informações, são as principais alterações do declínio da memória em decorrência do envelhecimento. A memória semântica é a mais estável no ato de envelhecer, pois está atrelada às informações linguísticas e à experiência educacional (Mellendez et al., 2018; Porto \& Nitrini, 2014; Yassuda \& Abreu, 2013).

Por outro lado, há muitas queixas, por parte dos idosos, relacionadas à dificuldade de lembrar eventos ou fatos que ocorreram recentemente, o que se refere à memória episódica. As queixas relacionam-se principalmente à falha da codificação e à recuperação, sem interferência no processo de armazenamento da informação (Porto \& Nitrini, 2014). Outro ponto comum dentre as dificuldades mnemônicas referidas pelos idosos é em relação à memória prospectiva, principalmente entre os idosos de idade mais avançada (Abrisqueta-Gomez, 2013; Lee et al., 2017; Mascarello, 2013).
As teorias da cognição social consideram o ser humano como um agente ativo sobre as circunstâncias e o ambiente que o circunda, bem como sobre o seu próprio comportamento, suas ações e emoções. O homem molda o seu ambiente e comportamento de forma ativa, não se limitando a ser apenas reativo aos estímulos externos. A concretização desses princípios de adaptação realiza-se por meio da autorreflexão e autorregulação, isto é, observando e pensando sobre as suas ações, sentimentos e cognições, avaliando o impacto das suas ações, definindo objetivos e implementando planos para alcançá-los (Payne et al., 2012).

Nessa perspectiva da relação entre indivíduo e ambiente, destaca-se a autoeficácia, que pode ser definida como a crença que o indivíduo tem sobre sua capacidade de realizar com sucesso determinada atividade. Dessa forma, sua crença pode afetar suas escolhas e o desempenho profissional (Payne et al., 2012; Rabelo, 2005). O conceito de autoeficácia descreve estruturas e funções que orientam a ação de uma pessoa sobre si mesma e sobre o ambiente (Bandura, 2008). Nesse sentido, os processos cognitivos permitem a mediação das experiências por meio dos sistemas sensório-motor e cerebral, os quais possibilitam ações com sentido e direção que proporcionam mais satisfação na vida (Benites et al., 2006; Motes et al., 2018).

A autoeficácia percebida é o mecanismo mais central e mais difundido na mediação entre o indivíduo e o meio ambiente, pois somente com esse tipo de crença o indivíduo é capaz de comportar-se ativamente, com motivação. A autoeficácia é a crença sobre as habilidades pessoais de organizar e executar alguma atividade. $\mathrm{O}$ seu desempenho pode ser alto, em pessoas que acreditam que são eficazes mesmo nas tarefas mais difíceis, ou baixo, naquelas que pensam ser capazes de desempenhar somente tarefas mais fáceis. Assim, esses tipos de desempenhos podem afetar consideravelmente a plasticidade do sistema nervoso, a capacidade de monitoramento e a modulação da cognição, da emoção e do comportamento (Araujo Assunção \& Chariglione, 2020).

O senso de autoeficácia pode variar de acordo com a complexidade da tarefa, exigindo do indivíduo maior ou menor esforço para desempenhá-la. Na literatura, existem autores como Benites et al. (2006) e West et al. (2008) que estudam a autoeficácia em domínios específicos como o autocuidado, a memória, o treinamento cognitivo, as relações interpessoais, as atividades instrumentais, entre outros, avaliados por meio de testes e tarefas específicas que podem inferir medidas de desempenho relacionados a este constructo.

Para Bandura (2008), julgamentos sobre as competências alheias são construções sociais que servem para classificar os indivíduos em rótulos, estereótipos e estigmas. Por exemplo, a relação entre declínio de memória e avanço da idade é um estigma social intimamente relacionado com o idoso (Carneiro \& Falcone, 2013). Crenças pessoais e sociais formam um sistema recíproco, de influência mútua. Os adultos que hoje possuem crenças generalizadas sobre 
a inabilidade dos idosos tenderão a ter baixa autoeficácia quanto às suas habilidades no futuro, quando farão parte da população de idosos (Benites et al., 2006; Carneiro \& Falcone, 2013).

Como existe um paradigma social estabelecido que a velhice acarreta um obrigatório declínio no desempenho físico e cognitivo, as instituições sociais se antecipam e criam barreiras à participação social dos idosos antes mesmo que eventuais incapacidades ou deficits se instalem. Segundo Neri (2006), os indivíduos criam suas expectativas de acordo com suas crenças e, neste contexto, consolidam paradigmas sobre a velhice, como a do idoso perder competência, e passam a se comportar conforme suas crenças.

Portanto, percebe-se que crenças de autoeficácia influenciam o modo como as pessoas sentem, pensam e agem. Uma baixa autoeficácia pode estar associada a depressão, ansiedade, sentimento de impotência, baixa autoestima e pensamentos mais pessimistas quanto às habilidades pessoais (Azzi \& Polydoro, 2006; Bourscheid et al., 2016; Gecas, 2003). Ainda segundo os autores, o nível de autoeficácia pode, desse modo, aumentar ou impedir a motivação e afetar, direta e indiretamente, a adaptação e a mudança. As crenças de autoeficácia podem determinar também o estabelecimento de objetivos, a escolha de atividade e a disposição para realizar esforços e persistir em tarefas. Além disso, as diferenças de idade, gênero e ambiente também são grandes influenciadores no desenvolvimento e na expressão da autoeficácia.

Assim, diante do referencial apresentado, este estudo teve como objetivo verificar o desempenho dos idosos na habilidade cognitiva da memória, bem como na percepção subjetiva da autoeficácia da memória. Outro ponto foi comparar o relato da queixa da falha de memória com a medida de desempenho, no intuito de confirmar a presença ou não dos estereótipos sociais em relação ao desempenho de memória dos idosos.

\section{MÉTODO}

O estudo foi desenvolvido em dois Centros de Convivência (CCI) e em quatro Unidades Básicas de Saúde (UBS), localizadas em três regiões administrativas do Distrito Federal. O delineamento caracterizou-se por uma pesquisa exploratória, quantitativa e transversal.

\section{Amostra}

A amostra por conveniência foi composta por 110 idosos, com média de idade igual a 70,25 \pm 7,65 anos (60-93anos), sendo $90 \%$ do sexo feminino; escolaridade igual a $69,1 \%$ com ensino fundamental incompleto e $12,7 \%$ com ensino médio completo, em um total médio de 5,72 $\pm 3,95$ anos de estudo. Foram estabelecidos como critérios de exclusão apresentar algum diagnóstico de transtorno cognitivo (transtorno cognitivo leve, demência, doença de Parkinson) ou negar-se a participar do estudo. $\mathrm{O}$ trabalho foi aprovado pelo Comitê de Ética da Universidade Católica de Brasília e pelo Comitê de Ética em Pesquisa FEPECS/SES-DF (20501513.3.0000.0029).

\section{Instrumentos}

Para a avaliação da memória, foi utilizado o Teste de Aprendizagem Auditivo-Verbal de Rey (RAVLT), um teste de medida breve que acessa a capacidade de memória imediata (memória de trabalho - A1), o índice de aprendizagem (somatória de A1 a A5), a evocação tardia livre (memória longo prazo - A7) e a capacidade de recordação (REC) (Magalhães \& Hamdan, 2010; Malloy-Diniz et al., 2007). A versão utilizada no presente estudo é a mesma do estudo de Lima (2007), sendo que a pontuação máxima das variáveis é de 15, exceto o índice de aprendizagem (A1 a A5), que é de 75.
Para a mensuração da autoeficácia, foram utilizados o Teste de Percepção Subjetiva da Memória (MAC-Q) e o Questionário de Memória Prospectiva e Retrospectiva (PRMQ-10). O MAC-Q é um instrumento de autoadministração, cujo objetivo é investigar como o indivíduo percebe a competência da sua memória. A pontuação máxima desse teste é 35 pontos e, quanto maior o valor, mais negativa é a percepção de falha da memória (Mattos et al., 2003). Quando o escore final é maior que 25 pontos, é considerado como percepção negativa da memória; de 15 a 25 pontos, o desempenho é considerado como dificuldades da memória; abaixo de 15 pontos, considera-se que o indivíduo não apresenta percepção de dificuldade da memória.

O teste PRMQ-10 objetivou avaliar o autorrelato de falhas de memória prospectiva e retrospectiva. Os pontos máximo e mínimo são 50 e 10, respectivamente, com ponto de corte 20 , visto que os indivíduos que apresentam pontuação igual ou acima de 20 apresentam alta queixa de falha de memória (Benites \& Gomes, 2007). Por sua vez, aqueles com pontuação abaixo de 20 são classificados como pessoas que não apresentam queixa de falha de memória.

\section{Procedimento metodológico}

Tendo em vista o delineamento para recrutar participantes idosos, inicialmente foi realizada uma palestra sobre Envelhecimento e Cognição, que finalizava com a explanação da pesquisa e o convite. Após o aceite do participante, houve contato para marcar o atendimento individual, com duração aproximada de 60 minutos para cada idoso. A sequência de aplicação dos instrumentos seguiu uma ordem, que compreendeu a leitura e assinatura 
do Termo de Consentimento Livre e Esclarecido (TCLE), o preenchimento do questionário sociodemográfico e a aplicação do instrumento Lista de Aprendizagem AuditivoVerbal de Rey, do teste de percepção subjetiva da memória e do Questionário de Memória Prospectiva e Retrospectiva.

\section{Procedimento de análises de dados}

Inicialmente, foi realizada uma análise descritiva dos dados para caracterização da amostra e avaliação da normalidade, por meio dos testes Kolmogorov-Smirnov e Shapiro-Wilk. Os dados foram representados por: média, mediana, desvio padrão e quartis. Para as análises inferenciais do desempenho cognitivo e da percepção subjetiva da autoeficácia da memória e de queixas na falha de memória, foram utilizados os testes paramétricos ANOVA One-Way e Teste T-Student. Já para os dados não paramétricos, foi utilizado o teste de Kruskal-Wallis. O nível de significância adotado foi de $\mathrm{p} \leq 0,05$ e o programa estatístico Statistical Package for Social Science (SPSS), versão 22.0, devidamente registrado para o projeto.

\section{Resultados}

Os resultados serão apresentados inicialmente pela caracterização sociodemográfica da amostra para o entendimento dos idosos analisados e, posteriormente, pelas medidas de memória e seus subtipos: memória imediata, índice de aprendizagem, evocação tardia, capacidade de recordação, percepção subjetiva da memória, memória prospectiva e retrospectiva. No que se refere à caracterização da amostra, em suas medidas de sexo, região administrativa e estado civil, este estudo apresentou a distribuição apresentada na Tabela 1.

Para além das informações citadas na Tabela 1, observouse que a média de anos de estudo foi de 5,72 anos $( \pm 3,95)$, apresentando o tempo mínimo de educação formal de um ano e tempo máximo de 22 anos. Por sua vez, em relação à profissão, 69,1\% dos idosos eram aposentados, seguidos por 16,4\% beneficiários (pensão). Por fim, 90,9 \% (100) dos idosos faziam uso regular de medicamentos, 30,0 \% apresentavam hipertensão arterial sistêmica, 14,4\% hipertensão arterial sistêmica e diabetes mellitus e 11,8 \% hipertensão arterial sistêmica e dislipidemia.

$\mathrm{Na}$ análise estatística pelo teste de Levene, os subconjuntos do RAVLT mostraram homogeneidade, não apresentando diferença significativa entre eles, visto que a variável idade não revelou interferência no desempenho das tarefas do RAVLT (Tabela 2). Os valores de significância foram: $\mathrm{A} 1 \operatorname{com} \mathrm{p}=0,30 ; \mathrm{A} 2 \operatorname{com} \mathrm{p}=0,11 ; \mathrm{A} 3 \operatorname{com} \mathrm{p}=0,74$; $\mathrm{A} 4$ com $\mathrm{p}=0,74$; $\mathrm{A} 5$ com $\mathrm{p}=0,16$; lista $\mathrm{B}$ com $\mathrm{p}=0,43$; $\mathrm{A} 6$ com $p=0,46$; A 7 com $p=0,49$; A1A5 com $p=0,09$; e REC com $\mathrm{p}=0,25$.

Nesse sentido, a variável do valor de aprendizagem mnemônica (somatória dos pontos de A1 a A5) apresenta valores crescentes em todos os grupos etários, o que confere a capacidade de aprendizagem preservada (Figura 1). Os dados da média dos idosos de 60 a 69 anos foi de 37,97 $( \pm 9,78)$, os de 70 a 79 anos foi de $35,0( \pm 9,03)$ e dos idosos acima de 80 anos foi de 29,31 $( \pm 7,26)$. No processo de

Tabela 1

Caracterização Sociodemográfica

\begin{tabular}{|c|c|c|}
\hline Variável & $\mathbf{N}$ & $\%$ \\
\hline \multicolumn{3}{|l|}{ Sexo } \\
\hline Homens & 99 & $90,00 \%$ \\
\hline Mulheres & 11 & $10,00 \%$ \\
\hline TOTAL & 110 & $100,00 \%$ \\
\hline \multicolumn{3}{|l|}{ Região Administrativa } \\
\hline Santa Maria & 57 & $51,80 \%$ \\
\hline Taguatinga & 18 & $16,40 \%$ \\
\hline Ceilândia & 8 & $7,30 \%$ \\
\hline Riacho Fundo & 8 & $7,30 \%$ \\
\hline Demais regiões* & 10 & $17,2 \%$ \\
\hline TOTAL & 110 & $100,00 \%$ \\
\hline \multicolumn{3}{|l|}{ Estado Civil } \\
\hline Casados & 43 & $39,10 \%$ \\
\hline Viúvos & 36 & $32,70 \%$ \\
\hline Solteiros & 18 & $16,40 \%$ \\
\hline Separados & 13 & $11,80 \%$ \\
\hline TOTAL & 100 & $100,00 \%$ \\
\hline
\end{tabular}

Nota: *Águas Claras, Vicente Pires, Samambaia, Guará e Brazlândia 
Tabela 2

Resultados do RAVLT*

\begin{tabular}{|c|c|c|c|c|c|c|}
\hline & \multicolumn{2}{|c|}{60 a 69 anos } & \multicolumn{2}{|c|}{70 a 79 anos } & \multicolumn{2}{|c|}{+80 anos } \\
\hline & Amostra & Referência & Amostra & Referência & Amostra & Referência \\
\hline A1 & $4,22( \pm 1,63)$ & $\begin{array}{c}5,7 \\
( \pm 1,4)\end{array}$ & $3,51( \pm 1,54)$ & $5,15( \pm 1,05)$ & $2,77( \pm 1,79)$ & $4,25( \pm 1,35)$ \\
\hline A2 & $6,52( \pm 2,02)$ & $8,0( \pm 1,65)$ & $6,23( \pm 2,46)$ & $\begin{array}{c}6,8 \\
( \pm 0,5)\end{array}$ & $5,23( \pm 1,69)$ & $5,9( \pm 1,4)$ \\
\hline A3 & $8,14( \pm 2,45)$ & $9,35( \pm 1,7)$ & $7,36( \pm 2,28)$ & $7,7( \pm 1,15)$ & $5,23( \pm 2,28)$ & $6,65( \pm 1,55)$ \\
\hline A4 & $9,31( \pm 2,53)$ & $10,5( \pm 1,65)$ & $8,79( \pm 2,17)$ & $9,15( \pm 1,25)$ & $7,54( \pm 2,07)$ & $7,85( \pm 1,55)$ \\
\hline A5 & $9,78( \pm 2,55)$ & $11,75( \pm 1,70)$ & $9,10( \pm 1,93)$ & $10,65( \pm 1,35)$ & $8,54( \pm 1,61)$ & $9,8( \pm 1,9)$ \\
\hline Lista B & $3,78( \pm 1,59)$ & $4,65( \pm 1,05)$ & $3,61( \pm 1,84)$ & $\begin{array}{c}4,2 \\
( \pm 1,3)\end{array}$ & $3,15( \pm 1,67)$ & $3,3( \pm 1,65)$ \\
\hline A6 & $8,10( \pm 2,74)$ & $10,4( \pm 2,0)$ & $7,18( \pm 2,39)$ & $8,9( \pm 1,25)$ & $5,38( \pm 1,71)$ & $8,05( \pm 2,1)$ \\
\hline A7 & $8,22( \pm 2,90)$ & $9,95( \pm 2,3)$ & $7,31( \pm 2,61)$ & $8,3( \pm 1,15)$ & $6,0( \pm 1,73)$ & $6,85( \pm 2,05)$ \\
\hline REC & $11,57( \pm 3,98)$ & $10,9( \pm 2,9)$ & $10,05( \pm 3,80)$ & $7,4( \pm 3,05)$ & $9,69( \pm 4,40)$ & $5,3( \pm 4,45)$ \\
\hline A1A5 & $37,97( \pm 9,78)$ & $45,35( \pm 6,55)$ & $35,00( \pm 9,03)$ & $39,45( \pm 3,95)$ & $29,31( \pm 7,26)$ & $34,45( \pm 5,95)$ \\
\hline
\end{tabular}

Nota: *RAVLT é a sigla do instrumento Lista de Aprendizagem Auditivo-Verbal de Rey

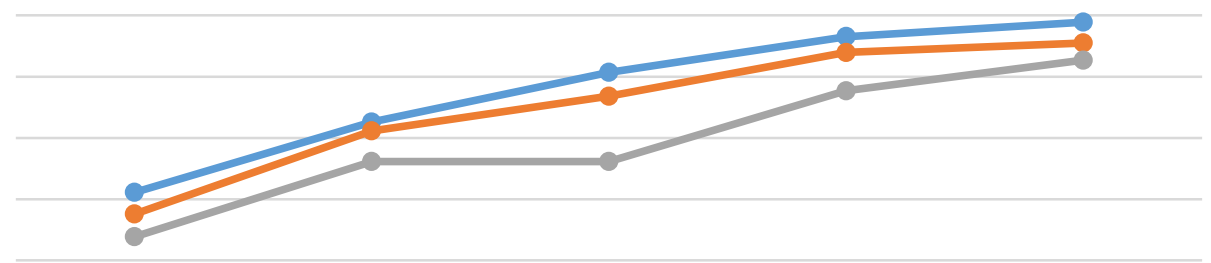

\begin{tabular}{|l|c|c|c|c|c|}
\cline { 2 - 6 } \multicolumn{1}{c|}{} & A1 & A2 & A3 & A4 & A5 \\
\hline $\mathbf{- 6 0}$ a 69 anos & 4,22 & 6,52 & 8,14 & 9,31 & 9,78 \\
\hline-70 a 79 anos & 3,51 & 6,23 & 7,36 & 8,79 & 9,1 \\
\hline- Acima de 80 anos & 2,77 & 5,23 & 5,23 & 7,54 & 8,54 \\
\hline
\end{tabular}

Figura 1. Índice de aprendizagem dos idosos, variável idade

Nota: Sendo as médias de A1 a A5 representativas dos diferentes momentos na curva de aprendizagem.

envelhecimento, isto é um indicativo fundamental, a respeito do funcionamento das habilidades cognitiva da capacidade da memória. Segundo Cotta et al. (2012), um desempenho normal no RAVLT é caracterizado pelo aumento de palavras memorizadas no decorrer das tarefas de A1 a A5, caracterizando uma curva de aprendizagem positiva.

Quando se avaliou as diferenças no índice de aprendizagem com relação à idade (A1 a A5), não foi observada diferença significativa entre os grupos $(p=0,09)$. Porém, pode-se perceber uma tendência a diferenças e acredita-se que isto ocorreria pelas diferenças no subgrupo A3 com relação à faixa etária acima de 80 anos.

Em relação aos valores do índice de aprendizagem e escolarização, observa-se que os piores desempenhos para aprendizagem foram apresentados pelos idosos com escolarização de 0 a 5 anos. Os idosos com mais de 11 anos de escolarização obtiveram o índice de aprendizagem de 64,62 , enquanto os indivíduos com grau de instrução de 1 a 5 anos obteve desempenho de 50,81.

Importante observar que, quando o desempenho da memória de trabalho (A1) é maior, a evocação tardia livre (A7) e o reconhecimento (REC) também apresentam resultados melhores, como evidenciado na Figura 2.

Outro ponto interessante é que os idosos da amostra apresentaram um desempenho bem inferior na subetapa A6, com média de 5,38 $( \pm 1,71)$, sendo $8,05( \pm 2,10)$ a média de referência para os idosos. Em compensação, na etapa subsequente (A7), há uma recuperação no desempenho dos sujeitos deste estudo, apresentando resultados similares ao de referência, com média de $6,00( \pm 1,73)$ para os idosos do estudo e média de $6,85( \pm 2,05)$ para os de referência. Ou seja, esses dados mostram que houve uma dificuldade na evocação 


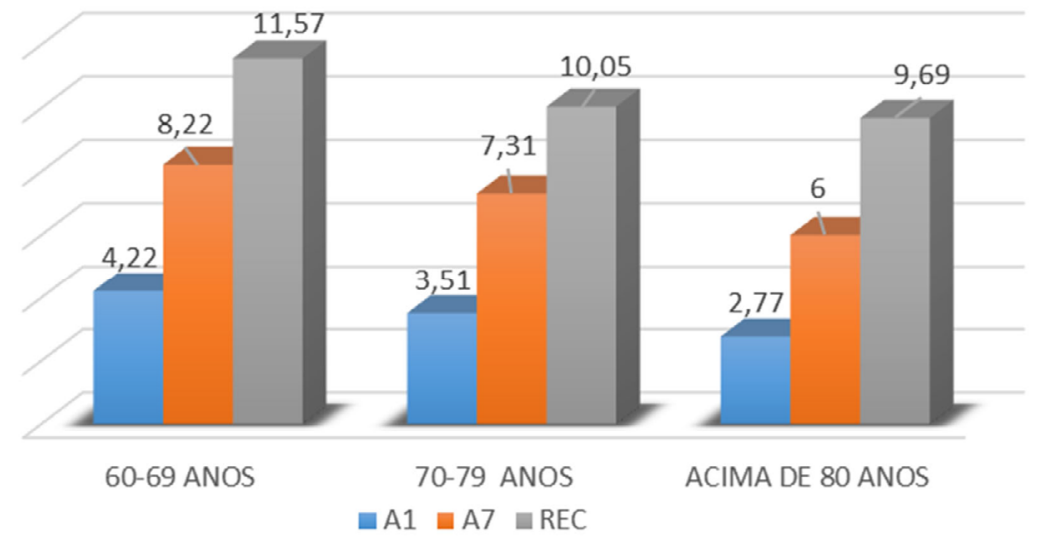

Figura 2. Desempenho da memória de trabalho, de longo prazo e reconhecimento Nota: A1 - memória imediata; A7 - memória de longo prazo; Rec - Recuperação.

da informação na memória a curto prazo, na qual a lista de distratores (lista B) pode ter influenciado no desempenho da recordação das palavras na etapa seguinte (A6). O dado corrobora a afirmativa de que a manipulação e seleção das informações necessárias dos distratores (fatores atencionais) podem interferir no processo de evocação da informação. Esse desempenho leva à reflexão de que não há efetivamente um deficit da memória propriamente dita. Se o problema fosse a dificuldade de memorizar as palavras, ou seja, um deficit somente da memória, os idosos não apresentariam melhora do desempenho na subtarefa $\mathrm{A} 7$, pois não se pode resgatar uma informação que não esteja memorizada.

Por fim, também foi evidenciado um melhor desempenho dos idosos na fase de reconhecimento, com média de $9,69( \pm 4,40)$, quando comparada com a referência, que é $5,3( \pm 4,45)$. Novamente, fica evidente que o estímulo foi armazenado/memorizado, mas há uma dificuldade em acessar essa informação, quando o idoso necessita de pistas externas para o melhor resgate da palavra memorizada.

Entre os idosos deste estudo, a média total de desempenho da percepção da autoeficácia da memória (MAC-Q) foi de $28,29( \pm 4,48)$, sendo o valor máximo igual 11,0 e o mínimo, 35,0 . Os resultados apresentam que $82,7 \%$ (91) dos idosos relataram uma percepção negativa da memória e somente $0,9 \%$ (1) apresentou uma percepção da memória boa. Outro dado relevante é que $99,10 \%$ (109) apresentaram alguma queixa subjetiva da memória. Na comparação de percepção subjetiva da memória e idade, os grupos foram homogêneos, tendo sido realizado o teste ANOVA, com o qual não foi verificada significância $(p=0,66)$. Em relação à educação formal, não houve relação com a percepção subjetiva da memória $(p=0,65)$, na qual os grupos apresentaram homogeneidade (teste ANOVA).

No questionário PRMQ - 10, a média geral dos idosos foi de 25,32 ( $\pm 10,59)$, sendo que $40,9 \%$ (45 idosos) relataram baixa queixa de memória e 59,1\% (65 idosos) obtiveram pontuação entre 20 e 50 pontos, o que caracteriza alta queixa de falha de memória. Em relação à idade e escolaridade, essas variáveis não interferiram na percepção de falha de memória entre os idosos deste estudo. Na análise estatística, os grupos apresentaram homogeneidade, de acordo com o teste ANOVA, sendo $p=0,03$ para a relação com a idade e $\mathrm{p}=0,22$, com a escolaridade.

Na memória retrospectiva, a média foi de 11,63 $( \pm 5,89)$, sendo que 49 idosos $(44,5 \%)$ relataram uma percepção de baixa falha da memória retrospectiva e $55,5 \%$ dos participantes referiram alta queixa de memória. Por outro lado, na memória prospectiva, a média do desempenho foi maior, de 13,69 ( $\pm 5,49)$, sendo que 32 idosos $(29,10 \%)$ declararam baixa queixa de memória e 78 indivíduos (70,9\%) apresentaram uma percepção de alta queixa da memória prospectiva.

\section{DISCUSSÃO}

Os idosos deste estudo apresentaram resultados do desempenho da memória episódica (RAVLT) que não indicam declínio nesta habilidade avaliada. Entretanto, é importante fazer algumas considerações dos desempenhos dos participantes deste estudo com os dados de referência. Tal fato pode ser elucidado por meio de pesquisas, como o estudo de Coelho et al. (2012), desenvolvido em Uberlândia-
MG com adultos e idosos, o qual verificou uma relação direta entre a escolaridade e os valores obtidos nos escores da avaliação cognitiva. Outro estudo também evidenciou a relação do desempenho de memória e a escolaridade, realizado no ambulatório de Geriatria da USP (HCFMUSP), com 20 idosos ativos, com média de 7,75 anos de ensino formal (Oliveira et al., 2014). 
$\mathrm{Na}$ avaliação da memória imediata e evocação tardia, a média da pontuação obtida nesse estudo foi inferior ao resultado encontrado no estudo de validação do teste RAVLT em mulheres brasileiras na faixa etária de 65 a 69 anos (Malloy-Diniz et al., 2007). Já a média do escore da memória de reconhecimento foi semelhante à pontuação obtida pela amostra do estudo de referência. Outro estudo realizado por Nespollo et al. (2017) com 29 idosas residentes de Cuiabá, participantes de um grupo de convivência e média de escolaridade de 7 anos, apresentaram escores superiores na memória imediata, evocação tardia e recordação livre.

A curva de aprendizagem de memória apresentouse crescente em todos os grupos etários, incluindo os octogenários, o que confere uma capacidade de aprendizagem preservada e pode ser um indicativo fundamental no processo do envelhecimento sobre a capacidade mnemônica. Alguns estudos referem que um desempenho normal no RAVLT, caracterizado pelo aumento de palavras memorizadas no decorrer das tarefas de A1 a A5, distinguindo uma curva de aprendizagem positiva (Cotta et al., 2012; Mattos et al., 2010; Nespollo et al., 2017).

Enfim, os indivíduos que frequentaram a educação formal por mais de 10 anos apresentaram os maiores valores para o índice de aprendizagem. Nesta perspectiva, pode-se inferir que a escolarização pode ser um fator protetivo no que se refere à aprendizagem e memória auditiva, no processo do envelhecimento.

Os estudos de Magalhães e Hamdan (2010) e MalloyDiniz et al. (2007) demonstraram que os idosos com alto grau de escolaridade obtiveram desempenho melhores nos escores da curva de aprendizagem, o que parece conferir que a escolaridade pode ter um enorme impacto sobre o desempenho do RAVLT e, por consequência, sobre a memória auditiva.

Em relação ao desempenho da memória de trabalho (A1), observou-se uma relação direta com os resultados da evocação tardia (A7) e do reconhecimento (REC). O estudo de Paula et al. (2012) também apresenta essa relação, o que reforça a importância da memória de trabalho no processo da memorização e, principalmente, na memória a longo prazo, no momento de resgate livre da informação. Em relação aos octogenários, observa-se um desempenho menor em relação à memória de trabalho, quando comparados com os idosos dos outros grupos etários, o que também refletiu de forma linear na diminuição do desempenho da evocação tardia livre da informação.

Vários estudos evidenciam que, com o avanço da idade, algumas habilidades cognitivas apresentam diminuição no desempenho, como atenção, função executiva e memória, sendo a última considerada a mais vulnerável no processo de envelhecimento (Chariglione et al., 2018; Chariglione et al., 2020; Coelho et al., 2012; Nespollo et al., 2017; Oliveira et al., 2014; Paula et al., 2012).

Dentre os resultados da percepção da autoeficácia da memória em idosos do presente estudo, a relevância se dá pelo fato de que $99,10 \%$ dessa população apresentaram alguma queixa subjetiva da memória, o que resultou na maioria relatar uma percepção de memória negativa. $\mathrm{Na}$ comparação da percepção subjetiva da memória com a idade e a escolaridade, não houve relação significante. Ou seja, as variáveis idade e educação formal não interferiram na percepção subjetiva da memória. No estudo de metanálise de autoeficácia e desempenho de memória, realizado por Beaudoin e Desrichard (2011), foi evidenciada uma queixa subjetiva da memória, principalmente entre a população idosa.

Outros estudos com a temática de autoeficácia também demonstraram uma relação inversa com a variável escolaridade (Guerreiro et al., 2006; Kim \& Suh, 2017), evidenciado no Projeto Potencial/Idade; a maioria dos idosos apresentava nível superior completo, mas a percepção subjetiva da memória mostrou-se negativa. Ou seja, o estudo não demonstrou uma relação com a escolaridade e diminuição da percepção negativa da memória, como foi observado na pesquisa de Bourscheid et al. (2016).

No estudo de Nascimento (2008), com idosos da cidade de Porto Alegre e Veranópolis, os resultados também demonstraram essa relação, onde a maioria dos idosos apresentou percepção negativa de autoeficácia, sendo que $41,5 \%$ dos idosos possuíam ensino fundamental completo; apesar do grau de escolaridade, não houve modificação da percepção negativa da queixa de memória. Portanto, os resultados desse estudo apresentam congruência com o desempenho dos idosos desta pesquisa, em que idade e escolaridade não foram variáveis que interferiram nos resultados da percepção subjetiva da memória. No geral, os dados sugerem que a grande maioria dos idosos apresentam uma percepção subjetiva da memória ruim, independentemente da idade e da escolaridade.

A autopercepção dos idosos revela que a maior queixa de falha da memória envolve a memória prospectiva em detrimento da retrospectiva, também evidenciado nos estudos de Benites e Gomes (2007). A justificativa para tal observação se alicerça na falha da habilidade atencional, ou seja, na execução das tarefas da memória prospectiva, que requerem a alocação de capacidade conscientes, em especial da atenção. A habilidade atencional, principalmente a atenção seletiva, é essencial nos processos de busca das pistas, sendo justamente o recrutamento das pistas (externas) necessário para o bom desempenho na memória prospectiva (Benites et al., 2006).

A dificuldade em detectar a pista pode refletir na redução da habilidade dos idosos em manter uma representação integrada do contexto da tarefa em um estado alto de ativação. É nessa etapa de seleção da pista que a memória de trabalho seria a responsável por essa redução, especialmente a etapa da codificação, que recruta os elementos da atenção.

Alguns autores argumentaram que o impacto da autoeficácia de memória e do desempenho da memória deve ser maior para os idosos do que para os adultos mais jovens, visto que os indivíduos mais jovens podem depender menos da capacidade da memória percebida ao fazer um teste de memória (Kim \& Suh, 2017; West et al., 2009). 
De acordo com Bandura (2008), quando os indivíduos começam a duvidar de sua capacidade de realizar uma tarefa, sua autoeficácia é reutilizada como um guia para a ação e, consequentemente, afeta seu desempenho na tarefa.

$\mathrm{Na}$ trajetória do envelhecimento, a questão de suas habilidades de memória se torna mais saliente, seja por maiores falhas de memória ou pela maior relevância pessoal do estereótipo de envelhecimento. Como resultado, os idosos podem realizar julgamentos de autoeficácia de forma mais sistemática do que os jovens, quando são confrontados com uma tarefa de memória.

Segundo Kim e Suh (2017), os resultados obtidos em relação à autoeficácia e ao desempenho da memória são inconsistentes, muito em virtude da dificuldade de se ter um alinhamento entre os estudos em relação aos instrumentos utilizados e à amostra.

\section{CONSIDERAÇÕES FINAIS}

Mediante os resultados, observa-se que o tipo de memória mais comprometido com o envelhecimento é a memória de curto prazo, especialmente a memória de trabalho. Apesar da diminuição da eficiência dos idosos na memória de trabalho, no momento necessário de reter e codificar a informação, as pessoas idosas, inclusive os idosos acima de 80 anos, mantêm a habilidade para adquirirem novas informações e estratégias de aprendizagem, como foi evidenciado no índice de aprendizagem de memória.

Outro aspecto que merece atenção, no envelhecimento ativo, é a percepção subjetiva das falhas de memória (metamemória) e a autoeficácia da memória, que demonstram que podem sofrer influência do meio sociocultural no qual o sujeito está inserido. Assim, se pode inferir a ação do ambiente nas crenças, nos estigmas e nos paradigmas sobre os indivíduos idosos, evidenciado pelos altos índices da percepção negativa da autoeficácia da memória, referida pelos participantes deste estudo.

Por outro lado, na avaliação da memória, os idosos apresentaram desempenhos dentro do intervalo de normalidade e, em algumas situações, específicos com desempenho superior, como no caso do reconhecimento da informação com pistas. Ou seja, a percepção negativa da memória muito provavelmente não se justifica pela habilidade da memória propriamente dita, porém muito mais pelos paradigmas socioculturais que ainda estão atrelados de forma tão negativa à pessoa idosa e ao processo de envelhecimento. Isso porque as crenças e os estigmas mais negativos sobre a memória podem ser variáveis moduladoras importantes do desempenho, tais como o esforço desprendido, motivação e uso de estratégias.

Enfim, é importante desmistificar constructos sociais de que o envelhecimento é sinônimo somente de um processo progressivo de declínio e, principalmente, que a pessoa idosa perde a sua capacidade de memorização e de aprendizagem. Os resultados deste estudo traduzem que o idoso preserva ao longo da vida a habilidade de aprender. Por fim, é preciso pensar em ações e políticas públicas no sentido de transformar paradigmas acerca da pessoa idosa no sentido de valorizar e, por que não, potencializar as capacidades que o idoso apresenta ao longo da vida.

A habilidade da memória e a autoeficácia em idosos são temas bastante complexos, sugerindo-se que estudos posteriores nesta população sejam desenvolvidos com uma amostra maior e com a manipulação de outras variáveis de desempenho e de desfechos, com a finalidade de se obter maiores reflexões acerca da temática.

\section{REFERÊNCIAS}

Abrisqueta-Gomez, J. (2013) Memória e envelhecimento cognitivo saudável. In L. F. Malloy-Dinniz, D. Fuentes, \& Cosenza, R. M. (Eds.), Neuropsicologia do envelhecimento (pp. 171-196). Artmed.

Araujo Assunção, J. L., \& Chariglione, I. P. F. S. (2020). Envelhecimento Cognitivo, Autoeficácia e Atividade Física: Uma Revisão Sistemática. Revista de Psicologia da IMED, 12(1), 116-132. doi:https://doi.org/10.18256/2175-5027.2020. v12i1.3120

Azzi, R. G., \& Polydoro, S. A. (2006). Autoeficácia em diferentes contextos. Alínea.

Bandura, A. (2008). Teoria Social Cognitiva: Conceitos básicos. Artmed.

Baumgart, M., Snyder, H. M., Carrillo, M. C., Fazio, S., Kim, H., \& Johns, H. (2015). Summary of the evidence on modifiable risk factors for cognitive decline and dementia: A population-based perspective. Alzheimer's \& Dementia, 11, 718-726. https://doi. org/10.1016/j.jalz.2015.05.016
Beaudoin, M., \& Desrichard, O. (2011). Are Memory SelfEfficacy and Memory Performance Related? A meta-analysis. Psychological Bulletin, 137(2), 211-241 https://doi.org/10.1037/ a0022106

Belleville, S., Hudon, C., Bier, N., Brodeur, C., Gilbert, B., Grenier, S., .Gauthier, S. (2018). MEMO+: Efficacy, Durability and Effect of Cognitive Training and Psychosocial Intervention in Individuals with Mild Cognitive Impairment. J Am Geriatr Soc. https://doi.org/10.1111/jgs. 15192

Benites, D., Gomes, W. B. (2007). Tradução, adaptação e validação preliminar do Prospective and Retrospective Memory Questionaire (PRMQ). Psico-USF, 12(1), 45-54. https://doi. org/10.1590/S1413-82712007000100006

Benites, D., Jacques, S.M.C., Gauer, G., \& Gomes,W. B. (2006). Percepção de auto-eficácia e auto-relato de falhas de memória prospectiva e retrospectiva. Interação em Psicologia, 10(2), 207-215. http://dx.doi.org/10.5380/psi.v10i2.7677

Boller, B., Mellah, S., Ducharme-Laliberté, G., \& Belleville, S. (2017). Relationships between years of education, regional grey 
matter volumes, and working memory-related brain activity in healthy older adults. Brain Imaging and Behavior, 11(2), 304317. https://doi.org/10.1007/s11682-016-9621-7

Bourscheid, F. R., Mothes, L., \& Irigaray, T. Q. (2016). Memória em idoso: Relação entre percepção subjetiva e desempenho em testes objetivos. Estudos de Psicologia I, 33(1),151-159. http:// dx.doi.org/10.1590/1982-027520160001000015

Burke, S. L., Hu, T., Fava, N. M., Li, T., Rodriguez, M. J., Schuldiner, K. L., Burgess, A., \& Laird, A. (2018). Sex differences in the development of mild cognitive impairment and probable Alzheimer's disease as predicted by hippocampal volume or white matter hyperintensities. Journal of Women \& Aging. doi: 10.1080/08952841.2018.1419476

Cançado, F. A., Alanis, L.M., \& Horta, M. L. (2013). Envelhecimento cerebral. In E.V. \& L. Py. (Eds.), Tratado de Geriatria e Gerontologia (3a ed., pp. 232-255). Guanabara Koogan.

Carneiro, R. S., \& Falcone, E. M. O. (2013). O desenvolvimento das habilidades sociais em idosos e sua relação na satisfação com a vida. Psicologia em Estudo, 18(3). http://dx.doi.org/10.1590./ S1413-294X2013000300012

Chariglione, I. P.F. (2014). Intervenções Cognitivas para o aprimoramento da memória em idosos com envelhecimento cognitivo normal [Tese de doutorado, Universidade de Brasília]. Repositório UnB. https://repositorio.unb.br/ handle/10482/16137

Chariglione, I. P. F. S., Janczura, G. A., \& Belleville, S. (2018). Cognitive interventions to improve memory in healthy older adults: The use of Canadian (MEMO) and Brazilian (Stimullus) approaches. Estudos de Psicologia (Natal), 23(1), 2-13. http:// dx.doi.org/10.22491/1678-4669.20180 002

Chariglione, I. P. F. S., Silva, H. S., Melo, G. F., Silva, K. H. C. V., \& Oliveira, M. L. C. (2020). Cognitive interventions and performance measures: a longitudinal study in elderly women. Estudos de Psicologia (Campinas), 37, e190032. Epub January 27, 2020.https://doi.org/10.1590/1982-0275202037e190032

Cromartie J. (2020). Demographic components of aging in the nonmetropolitan U.S., 1980-2017. In: Glick J., McHale S., King V. (eds) Rural Families and Communities in the United States. National Symposium on Family Issues, (pp. 145-166). vol 10. Springer, Cham

Coelho, F. G. M, Vital, T. M., Novais, I. P., Costa, G. A., Stella, F., Santos-Galduroz, R. F. (2012). Desempenho cognitivo em diferentes níveis de escolaridade de adultos e idosos ativos. Rev Bras Geriatr Gerontol, 15(1), 7-15. https://doi.org/10.1590/ S1809-98232012000100002.

Cotta, M. F., Malloy-Diniz, L. F., Nicolato, R., Moares, E. N., Rocha, F. L., \& Paula, J. J. (2012). O Teste de Aprendizagem Auditivo-Verbal de Rey (RAVLT) no diagnóstico diferencial do envelhecimento cognitivo normal e patológico. Contextos Clínicos, 5(1),10-25. doi: 10.4013/ctc.2012.51.02

Gecas, V. (2003). Self-Agency and the life course. In J. T. Mortimer \& M. J. Shanahan (Eds.), Handbook of the life course (369388). Kluwer Academic/Plenum Publishers.

Guerreiro, T. C., Veras, R., Motta, L. B., Veronesi, A. S., \& Schmidt, S. (2006). Queixa de memória e disfunção objetiva de memória em idosos que ingressam na oficina de memória na UnATI. Rev Bras Geriat. Gerontol, 9(1), p.7-20. http://saudepublica.bvs. $\mathrm{br} /$ pesquisa/resource/pt/lil-450304

Kim, E. H., \& Suh, S. R. (2017). Effects of a Memory and VisualMotor Integration Program for Older Adults Based on SelfEfficacy Theory. J Korean Acad Nurs, 47(3), 431-444. doi. org/10.4040/jkan.2017.47.3.431

Lee, S. D., Ong, B., Pike, K. E., \& Kinsella, G. J. (2017). Prospective memory and subjective memory decline: A neuropsychological indicator of memory difficulties in community-dwelling older people. J Clin Exp Neuropsychol, 40(2), 183-197. doi: 10.1080/13803395.2017.1326465
Lima, J.S. (2007). Efeitos do treino de memória e da atividade física em portadores da doença de Alzheimer (Dissertação de Mestrado não publicada). Universidade Federal de Santa Catarina.

Magalhães, S. S., \& Hamdan, A. C. (2010). The Rey Auditory Verbal Learning Test: Normative data for the Brazilian population and analysis of the influence of demographic variables. Psychology \& Neuroscience, 3(1), 85-91. doi: 10.3922/j.psns.2010.1.011

Malloy-Diniz, L. F., Lasmar, V. A. P., Gazinelli, L. S. R., Fuentes, D., \& Salgado, J. V. (2007). The Rey Auditory-Verbal Learning test: Applicability for the Brazilian erderly population. Rev. Bras Psiquiatria, 29(4), 324-329. http://dx.doi.org/10.1590/ S1516-44462006005000053

Mascarello, L. J. (2013). Memória de trabalho e processo de envelhecimento. Psicologia Revista, 22(1), 43-59. https:// revistas.pucsp.br/index.php/psicorevista/article/view/16657

Mattos, P., Lino, V., \& Rizo, L., Alfano, A. Araújo, K. \& Raggio, R. (2003). Queixas de memória de idosos saudáveis e desempenho em testes. Arquivos de Neuropsiquiatria, 61(4), 920-24. http:// www.scielo.br/pdf/anp/v61n4/a06v61n4.pdf

Meléndez, J. C., Agusti, A., Satorres, E., \& Pitarque, A. (2018). Are semantic and episodic autobiographical memories infuenced by the life period remembered? Comparison of young and older adults. Eur J Ageing, 1-8. https://doi.org/10.1007/ s10433-018-0457-4.

Melo, B. R. S., Diniz, M. A. A., Casemiro, F. G., Figueiredo, L. C., Santos-Orlandi, A. A., Haas, V. J., Orlandi, F. S., \& Gratão, A. C. M. (2017). Avaliação cognitiva e funcional de idosos usuários do serviço público de saúde. Esc Anna Nery, 21(4), e20160388. http://dx.doi.org/10.1590/2177-9465EAN-2016-0388

Miranda, G. M. D, Mendes, A. C. G., \& Silva, A. L. A. (2016) $\mathrm{O}$ envelhecimento populacional brasileiro: Desafios e consequências sociais atuais e futuras. Rev. Bras. Geriatr. Gerontol, 19(3),507-519. http://dx.doi.org/10.1590/180998232016019.150140

Motes, M. A., Yezhuvat, U. S., Aslan, S., Spence, J. S., Rypma, B., \& Chapman, S. B. (2018). Higher-order cognitive training effects on processing speed-related neural activity: A randomized trial. Neurobiology of Aging, 62, 72-81.

Nascimento, N. M. R. (2008). Estudo comparativo sobre a prevalencia de declínio cognitivo entre dois grupos de idosos (Dissertação de Mestrado não publicada). PUC-Rio Grande do Sul.

Neri, A. L. (2006). O senso da auto-eficácia como mediador do envelhecimento bem sucedido no âmbito da cognição, das competências para a vida diária e auto cuidado. In R. G. Azzi \& S. A. Polydoro (Eds.), Autoeficácia em diferentes contextos (pp. 59-85). Alínea.

Nespollo, A. M., Marcon, S. R., Lima, N. V. P., Dias, T. L., \& Espinosa, M. M. (2017). Condições de saúde e desempenho da memória: Um estudo com idosas. Rev. Bras. Enfem., 70 (3),668-74. http://dx.doi.org/10.1590/0034-7167-2016-0529

Oliveira, R. S, Trezza, B. M., Busse, A. L., \& Jacob-Filho, W. (2014). Efeito de aprendizagem de testes cognitivos computadorizados em idosos. Einstein, 12(2),149-153. doi: 10.1590/S1679-45082014AO2954

Paula, J. J., Melo, L. P. C., Nicolato, R., Moraes, E. N., Bicalho, M. A., Hamdan, A. C., \& Malloy-Diniz, L. F. (2012). Fidedignidade e validade de construto do Teste de Aprendizagem AuditivoVerbal de Rey em idosos brasileiros. Rev Psiq Clín., 39(1),1923. https://doi.org/10.1590/S0101-60832012000100004

Payne, B. R., Jackson, J. J., Hill, P. L., Gao, X., Roberts, B. W., \& Stine-Morrow, E. A. L. (2012). Memory self-efficacy predicts responsiveness to inductive reasoning training in older adults. The Journals of Gerontology, Series B: J Gerontol B Psychol Sci Soc Sci., 67(1), 27-35. doi:10.1093/geronb/gbr073 
Porto, F. H. G, \& Nitrini, R. (2014). Neuropsicologia do envelhecimento normal e do comprometimento cognitivo leve. In L.Caixeta \& Al. L.Teixeira (Eds.), Neuropsicologia Geriátrica I (pp. 141-152). Artmed.

Rabelo, D.F. (2005). Auto-eficácia. In A. L. Neri (Ed.), Palavraschave em Gerontologia (pp.19-22). Alínea.

Ribeiro, A. I., Krainski, E. T., Carvalho, M. S., Launoy, G., Pornet, C., \& Pina, M. F. (2018). Does community deprivation determine longevity after the age of 75 ? A cross-national analysis. Int. J Public Health. https://doi.org/10.1007/s00038018-1081-y

Tomaszewski Farias, S., Giovannetti, T., Payne, B., Marsiske, M., Rebok, G., Schaie, K., Gross, A. (2018). Self-perceived Difficulties in Everyday Function Precede Cognitive Decline among Older Adults in the ACTIVE Study. Journal of the International Neuropsychological Society, 24(1), 104-112. doi:10.1017/S1355617717000546
West, R. L., Bagwell, D. K., \& Dark-Freudeman, A. (2008). Self-Efficacy and Memory Aging: The Impact of a Memory Intervention Based on Self-Efficacy, Aging, Neuropsychology, and Cognition: A Journal on Normal and Dysfunctional Development, 15(3), 302-329. http://dx.doi. org/10.1080/13825580701440510

West, R. L., Dark-Freudeman, A., \& Bagwell, D. K. (2009). Goalsfeedback conditions and episodic memory: Mechanisms for memory gains in older and younger adults. Memory, 17, 233-244. doi:10.1080/09658210802236407

Yassuda, M. S., \& Abreu, V. P. S. (2013). Avaliação Cognitiva do Idoso. In E. V. Freitas \& L. Py (Eds.), Tratado de Geriatria e Gerontologia (3a ed, pp. 2057-2067). Guanabara Koogan.

Yassuda, M. S., Viel, T. A., Silva, T. B. L., \& Albuquerque, M. S. (2013). Memória e envelhecimento: Aspectos cognitivos e biológicos. In E. V. Freitas \& L. Py (Eds.), Tratado de Geriatria e Gerontologia (3a ed., pp. 2046-2056). Guanabara Koogan. 